\title{
Multiply quantized vortices in trapped Bose-Einstein condensates
}

\author{
Emil Lundh \\ Department of Theoretical Physics, Umeå University, SE-901 87 Umeå
}

\begin{abstract}
Vortex configurations in rotating Bose-Einstein condensed gases trapped in power-law and anharmonic potentials are studied. When the confining potential is steeper than harmonic in the plane perpendicular to the axis of rotation, vortices with quantum numbers larger than one are energetically favorable if the interaction is weak enough. Features of the wave function for small and intermediate rotation frequencies are investigated numerically.
\end{abstract}

\section{INTRODUCTION}

Trapped Bose-Einstein condensed gases provide a novel kind of condensed matter system which can sustain quantized vortices, as has been realized in the recent years [1, 2, 3, 1. Features which differ from those in macroscopic superfluid systems are expected here. The fact that these systems are finite, and that the parameter regime where the vortex core size is comparable to the system size is attainable, in principle opens up for the possibility for vortices in these systems to have a circulation quantum number $q$ larger than unity.

It is well known that multiply quantized vortices are not thermodynamically stable in spinless, macroscopic and homogeneous superfluids, because the energy of a vortex depends on the square of the circulation, and therefore two singly quantized vortices with a finite spatial separation have lower energy than one doubly quantized, while giving the system the same angular momentum [5]. However, multiply quantized vortices may well be energetically favorable in systems which do not fulfill the criteria of being homogeneous, spinless and macroscopic. The assumption of homogeneity is not met in superconductors with pinning forces, where indeed doubly quantized vortices are observed [6]. In ${ }^{3} \mathrm{He}-\mathrm{A}$, where the order parameter is not a scalar due to spin degrees of freedom, a lattice of doubly quantized vortices with filled cores has recently been observed [7]. The argument also fails in spatially confined systems where the vortex cores are not much smaller than the system, such as mesoscopic superconducting disks, where vortices with large quantum numbers have been predicted [8].

In condensed Bose gases confined in harmonic-oscillator potentials, it has been found both analytically [9], numerically [10, 11] and variationally [11] that multiply quantized vortices are not energetically favorable, and no quantum numbers larger than unity have been observed in experiment [3, 4. However, none of these studies has considered whether altering the power law of the confining potential can open up for the existence of multiply quantized vortices. In this paper, we shall give rigorous criteria for the thermodynamic stability of multiply quantized vortices in trapped Bose-Einstein condensates, and show the decisive role played by the shape of the potential.

The system under study is a gas of bosons of mass $m$ in an external potential $V(r)$, dilute enough and at sufficiently low temperature that it is well described by the Gross-Pitaevskii equation [12, 13, 14]

$$
i \hbar \frac{\partial \psi(r, t)}{\partial t}=-\frac{\hbar^{2}}{2 m} \nabla^{2} \psi(r, t)+V(r) \psi(r, t)+U_{0}|\psi(r, t)|^{2} \psi(r, t)-\Omega \hat{L_{z}} \psi(r, t) .
$$

The so-called condensate wave function $\psi(r, t)$ is normalized to the number of particles $N$ in three dimensions, and to the number of particles $\nu$ per unit length in two dimensions. The coefficient $U_{0}$ in front of the nonlinear term is the interaction strength, defined as $U_{0}=4 \pi \hbar^{2} a / m$, where $a$ is the s-wave scattering length. It turns out that the effective measure of interaction strength in two dimensions is the product $4 \pi \nu a$, which we shall denote by $g$. In three dimensions the corresponding quantity depends on the trapping potential.

A centrifugal term is present in the Gross-Pitaevskii equation, corresponding to a rotation of the trap with the frequency $\Omega$ about the $z$ axis. At certain critical values $\Omega_{\mathrm{c} q}$ of the rotation frequency, we expect there to be a discrete transition between states of different circulation numbers, so that when $\Omega_{\mathrm{c} q+1}>\Omega>\Omega_{\mathrm{c} q}$, a state with total circulation quantum number $q$ is the ground state [5]. The critical frequencies are functions of the coupling strength $U_{0}$ [15].

When the Gross-Pitaevskii equation is valid, the total energy of the system is given by the mean-field energy functional

$$
E=\int d r \psi^{*}(r)\left(-\frac{\hbar^{2}}{2 m} \nabla^{2}+V(r)-\Omega \hat{L}_{z}\right) \psi(r)+\frac{1}{2} U_{0}|\psi(r)|^{4} .
$$

We shall in the following be concerned with the lowest-energy solutions of the Gross-Pitaevskii equation (11) for a range of values of the driving frequency $\Omega$, interaction strength $U_{0}$ and for different external potentials $V$. The paper is organized as follows. In Section II, we present an analytic study of systems contained in three- and two-dimensional power-law and anharmonic traps. In Sec. III, we illustrate these findings numerically for the two-dimensional case 
and study some features of the different states with small and intermediate circulation quantum numbers. Sec. IV provides a conclusion.

\section{VORTEX CONFIGURATIONS IN POWER-LAW AND ANHARMONIC TRAPS}

Consider a Bose gas trapped in a cylindrically symmetric power-law potential of power $n$, which in cylindrical coordinates is written

$$
V(r, \phi, z)=\hbar \omega\left(\frac{r}{d_{t}}\right)^{n}+V_{\|}(z) .
$$

The trap frequency $\omega$, which determines the strength of the potential, defines a trap length $d_{t}=\sqrt{\hbar / m \omega}$. The dependence of the potential on the $z$ coordinate is totally arbitrary as long as the problem remains separable in the noninteracting limit, as is the case here. We shall hereafter neglect to mention the irrelevant $z$-dependent term and its associated degrees of freedom.

In the analysis of this section, we shall consider the weakly-interacting limit where $U_{0}$ is small, because if a multiply quantized vortex can ever be present, it will be so when the interaction is weak. To see why, note that the vortex cores are large for weak interactions and vice versa [14, 16. Therefore, in the limit of large $U_{0}$, the vortices are much smaller than the system size and the radius of curvature of the density profile. The result of a homogeneous bulk system thus applies, namely that a vortex array is the energetically favorable configuration. Therefore, we expect multiply quantized vortices to show up only in the realm of small (and possibly intermediate) $U_{0}$.

Ref. 9] showed why multiply quantized vortices are never the minimum-energy state in a system which is harmonically confined in the $x-y$ plane. We will here extend the same analysis to the case when the confinement is steeper than harmonic. The argument is based on perturbative treatment of the interaction term in the Gross-Pitaevskii equation (1). We therefore first study the noninteracting gas. When $U_{0}=0$, the Gross-Pitaevskii equation is identical to the one-particle Schrödinger equation, whose solutions are well known when the radial confinement is harmonic. The eigenstates $\varphi_{n_{r}}$ can be labeled by a radial and an axial quantum number $n_{r}$ and $q$ (as well as a quantum number associated with the $z$ direction, irrelevant for our considerations). The eigenenergies including the centrifugal term are

$$
E_{n_{r} q}=\hbar(\omega-\Omega) q+\hbar \omega\left(1+n_{r}\right)
$$

We shall concentrate on the states with no radial nodes, $\varphi_{0 q}$, because these have the lowest energy for a given angular momentum $N q \hbar$. These are in fact vortices with quantum number $q$. When the driving frequency $\Omega$ is less than the trap frequency $\omega$, the $q=0$ state is the ground state; when $\Omega=\omega$, all the states $\varphi_{0 q}$ are degenerate. When $\Omega>\omega, E$ decreases with increasing $q$, and there is no ground state. Hence, in the noninteracting case all the critical frequencies $\Omega_{\mathrm{c} q}$ are equal to the trap frequency $\omega$.

Following Ref. [9], we now consider perturbatively the interaction energy. The perturbation for a given wave function $\psi$ is, from Eq. (2),

$$
H^{\prime}=\frac{1}{2} U_{0} \int d r|\psi(r)|^{4} .
$$

Calculating this for general superpositions $\psi(r)=\sum_{q} C_{q} \varphi_{0 q}(r)$, we find that the degeneracy at $\Omega=\omega$ is lifted, and the critical frequencies $\Omega_{\mathrm{c} q}$ split apart and assume values less than $\omega$. Fixing a total angular momentum $L_{z}=N q \hbar$, it turns out that wave functions which are superpositions of states of different $q$ have less interaction energy than the pure multiply-quantized vortex configurations $\varphi_{0 q}$ for $q \geq 2$. Such superpositions are vortex arrays, and these are the energetically favorable states for any finite interaction strength.

We shall now show how the analysis of Ref. [9] works when the trapping power $n$ in Eq. (3) is larger than two. In this case, the single-particle energy varies faster than linear with the angular momentum. This implies that in the noninteracting case, pure multiply-quantized vortex states $\varphi_{0 q}$ have lower energy than any superposition having the same angular momentum. Hence, it takes a finite (albeit possibly very small) interaction strength $U_{0}$ to overcome this difference. We stress that in order to arrive at this result, we did not need to know any details of the eigenfunctions of a general power-law potential: the fact that the energy depends stronger than linearly on the angular momentum is enough. For a finite but weak enough interaction, the multiply-quantized vortex states are the minimum-energy configurations within their respective ranges of external rotation frequency $\Omega$. When the interaction is strong enough, however, the larger interaction energy of the multiply-quantized vortex states will overcome the difference in kinetic plus trap energy, and the ground state will again be a vortex array. This makes sense, since as we noted above, in the limit of strong interaction the results of the infinite and homogeneous case apply. 
For potentials weaker than harmonic, $n<2$, no rotation is possible. The potential in a frame rotating with angular frequency $\Omega$ is $V(r)-\frac{1}{2} \Omega r^{2}$, which is not confining if $\Omega>0$ and $V(r)$ is weaker than harmonic. Therefore a rotating state in such a trap can be at most metastable. 117.

If a pure power-law potential which is steeper than harmonic may seem an artificial construction which is hard to fabricate in practice, we note that the analysis can be repeated for an anharmonic potential,

$$
V(r, \phi, z)=\frac{1}{2} m \omega^{2} r^{2}\left(1+\lambda \frac{r^{2}}{d_{t}^{2}}\right)+V_{\|}(z) .
$$

The important point is that for any positive anharmonicity $\lambda$, the single-particle energy varies faster than linear with the angular momentum. This again leads to the conclusion that multiply quantized vortices can exist in anharmonic traps as long as $\lambda>0$ and the coupling is small.

We have shown that multiply quantized vortices can be stable only in trapping potentials which are steeper than harmonic in the $x-y$ plane, and only for weak enough interactions. A close inspection of the above argument reveals the physical mechanism behind the transition. The kinetic energy gives rise to a repulsion between the vortices because of the overlapping velocity fields, while the trapping potential strives to keep the vortices together in order to reduce the spatial extent of the cloud. The interaction energy favors states with several separated vortices because such states have lower density on the average. The thermodynamic stability of multiply quantized vortices is thus a consequence of the competition between these three energies, and the power 2 is the limiting value.

\section{NUMERICAL RESULTS FOR TWO-DIMENSIONAL SYSTEMS}

We now perform a numerical study to quantify the predictions of Section II. We shall treat the two-dimensional case only. We insert the power-law potential, Eq. (3), into the Gross-Pitaevskii equation (1) and scale out the dimensions by defining $r=d_{t} \tilde{r}, t=\omega^{-1} \tilde{t}, \psi=\sqrt{\nu} d_{t}^{-1} \tilde{\psi}, L_{z}=\hbar \tilde{L}_{z}, \Omega=\omega \widetilde{\Omega}, g=4 \pi \nu a$, and for the total mean-field energy $E=\hbar \omega \tilde{E}$. There results a dimensionless Gross-Pitaevskii equation,

$$
i \frac{\partial \tilde{\psi}}{\partial \tilde{t}}=-\frac{1}{2} \nabla_{\tilde{r}}^{2} \tilde{\psi}+\frac{1}{2} \tilde{r}^{n} \tilde{\psi}+g|\tilde{\psi}|^{2} \tilde{\psi}-\widetilde{\Omega} \hat{\tilde{L}}_{z} \tilde{\psi}
$$

which depends on three parameters, namely the coupling $g$, the rotation frequency $\widetilde{\Omega}$, and the trapping power $n$. We solve it in two dimensions using the split Fourier method [11. By propagating Eq. (7) in complex time, the system relaxes to a local energy minimum which depends on the initial state. In order to find the global minimum-energy configuration for each choice of parameters $g, \widetilde{\Omega}, n$, we perform the relaxation a few times using different, irregular initial states, compute their energy with the aid of Eq. (2), and out of the different final states we choose that which has the lowest energy. The grid size is $64 \times 64$ points, and the lattice constant is equal to 0.2 times the trap length $d_{t}$. We have confirmed that doubling the grid size and halving the lattice constant does not alter the results.

Figure 1 is a phase diagram for the quartic potential $(n=4)$, which shows how the $\widetilde{\Omega}-g$ plane is divided into regions of different vortex configurations with the total circulation $q$ ranging from 1 up to 4 . The different vortex configurations are labelled by the total number of quanta followed by the number of singularities present in the system: the configuration 3-3 thus denotes three $q=1$ vortices whereas 3-1 is a state with one triply quantized vortex present. The region marked by 0 is the nonrotating state. Since the numerical computation is done with limited precision, we need an operational criterion for the existence of a multiply quantized vortex. A $q$-fold quantized vortex is defined as a configuration in which the calculated circulation around a circle enclosing the origin is equal to $q$ and only one density minimum can be seen on optical inspection of the plotted wave function, i. e. the inter-vortex distance is less than the lattice constant of the numerical grid. The transition frequencies found using this criterion coincides with the point where the angular momentum saturates at an integer value.

As expected from the analysis of the previous section, we find multiply quantized vortices for small values of $g$, which split into arrays of singly quantized vortices for larger $g$. The transition from a multiply quantized vortex to an array is continuous, as illustrated in Figure 2, where two vortices are seen to merge as the critical line is crossed.

It is convenient to define a new quantity to describe the regime of stability of multiply quantized vortices. The $q$-th critical coupling $g_{\mathrm{c} q}$ is defined so that when $g>g_{\mathrm{c} q}$, there can exist no $q$-fold quantized vortices for any value of $\Omega$, but when $g<g_{\mathrm{c} q}$, a $q$-fold quantized vortex is the energy minimum within certain limits of $\Omega$. The critical coupling is a function of the trap power $n$. Fig. 1 suggests that the critical coupling increases with $q$. The physical reason for this is, that if the system is to accommodate many singly quantized vortices, the cores must be small and $g$ large. Our analysis in the previous section showed that $g_{\mathrm{c} q}=0$ for the harmonic trap, $n=2$, and $g_{\mathrm{c} q}>0$ when $n>2$. This is confirmed in Figure 3, which shows how $g_{\mathrm{c} 2}$ depends on $n$. The data points suggest that the curve $g_{\mathrm{c} 2}(n)$ goes to 


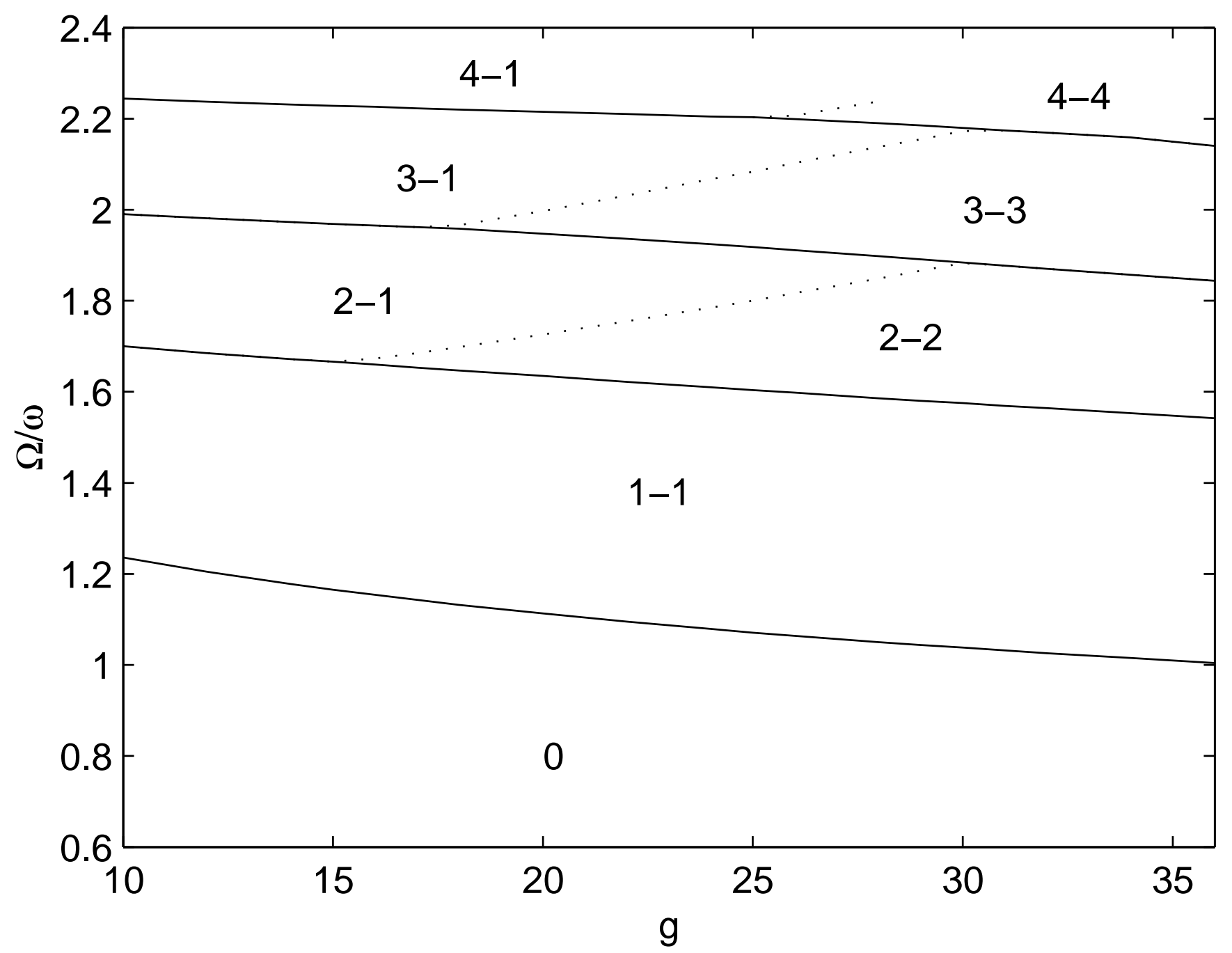

FIG. 1: Phase diagram for a quartically confined two-dimensional Bose gas subject to a force rotating with an angular frequency $\Omega$. The state marked by 0 is the nonrotating state, and $1-1$ is a state with one central $q=1$ vortex. The regions $2-2,3-3$ and 4-4 denote vortex lattices containing two, three and four singly quantized vortices, respectively, while the states $2-1,3-1$ and 4-1 are states with several quanta of circulation but only one phase singularity.

zero linearly when $n \rightarrow 2$ from above.

We have pursued the analogous anharmonic case, Eq. (6), using the same numerical procedure. The qualitative features of the phase diagram are not different from the pure power-law case. The critical coupling $g_{\mathrm{c} q}$ is now a function of the anharmonicity $\lambda$. The result for $g_{\mathrm{c} 2}$ is shown in Figure 1 . The limit $\lambda \rightarrow \infty$ is the quartic potential, whose critical coupling $g_{\mathrm{c} 2}=30.5$ can be read off from Fig. 11. When $\lambda \rightarrow 0$, on the other hand, the potential approaches a harmonic one, and $g_{\mathrm{c} 2}$ approaches zero. In the experimentally realized two-dimensional condensates of Ref. [18], $g$ varies between 100 and $10^{4}$, the former for a number of particles $N=10^{4}$. Diminishing the number of particles by a factor 10 and introducing an anharmonicity larger than 0.1 would take these systems below $g_{\mathrm{c} 2}$.

The phase diagram in $g$ - $\Omega$ space becomes richer for higher quantum numbers $q$. For $q \leq 7$, there are only two phases, a vortex-array phase and a multiply-quantized vortex phase. For higher quantum numbers, however, a new kind of pattern appears, where one multiply quantized vortex is surrounded by singly quantized vortices. Figure 5 shows the different vortex phases for $8 \leq q \leq 11$. The power of the potential is chosen to $n=2.2$. We find a number of distinct phases, which we label by their total circulation followed by the number of singularities just as in Fig. 1 . There are three phases containing eight quanta of circulation: a central $q=8$ vortex for small $g$ (denoted 8-1); an array of eight vortices for large $g(8-8)$; and for intermediate $g$, a central $q=2$ vortex surrounded by six $q=1$ vortices (8-7). The transition from 8-1 to 8-7 is continuous: the six $q=1$ vortices separate from the central one in a smooth way as the critical line is crossed. The two phases $8-7$ and $8-8$, on the other hand, have different symmetries and the 

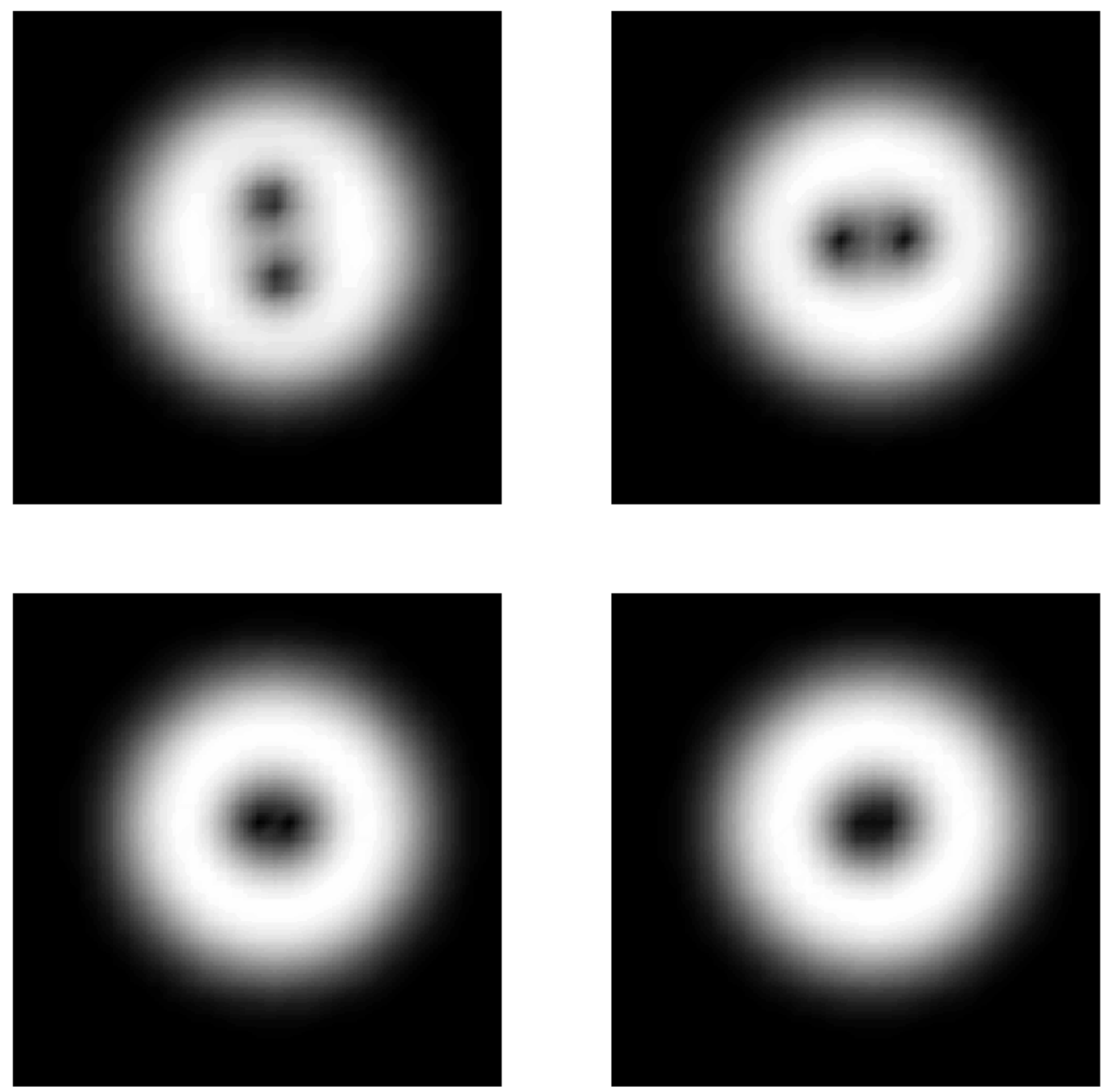

FIG. 2: Numerically computed condensate wave functions, illustrating how the distance between two vortices continuously decrease as the external rotation frequency $\Omega$ increases. The bright shades indicate high density and vice versa. The confinement is quartic, the coupling constant $g=28.0$, and $\Omega / \omega=1.59$ for the top left panel, 1.72 (top right), 1.83 (bottom left) and 1.86 (bottom right). The latter is the splitting frequency, operationally defined as the frequency where no mode of plotting the wave function reveals more than one density minimum.

transition between these two is discontinuous. For the case of nine quanta, there exist the corresponding phases 9-1 and 9-9, and one intermediate phase 9-8 with a central $q=2$ vortex and seven $q=1$ vortices. A 9-7 configuration with a $q=3$ vortex surrounded by $\operatorname{six} q=1$ vortices seems to be stable in a very narrow region of width $\Delta g \sim 0.2$, between 9-1 and 9-8, but with the present numerical precision we have not been able to accurately determine the boundaries of this phase and it is left out of Fig. 5. For the case $q=10$, on the other hand, one can clearly distinguish four different configurations, and likewise for $q=11$.

Analogously to the $q=8$ case, the transition between 9-1 and 9-7 is continuous, and so is the transition between 


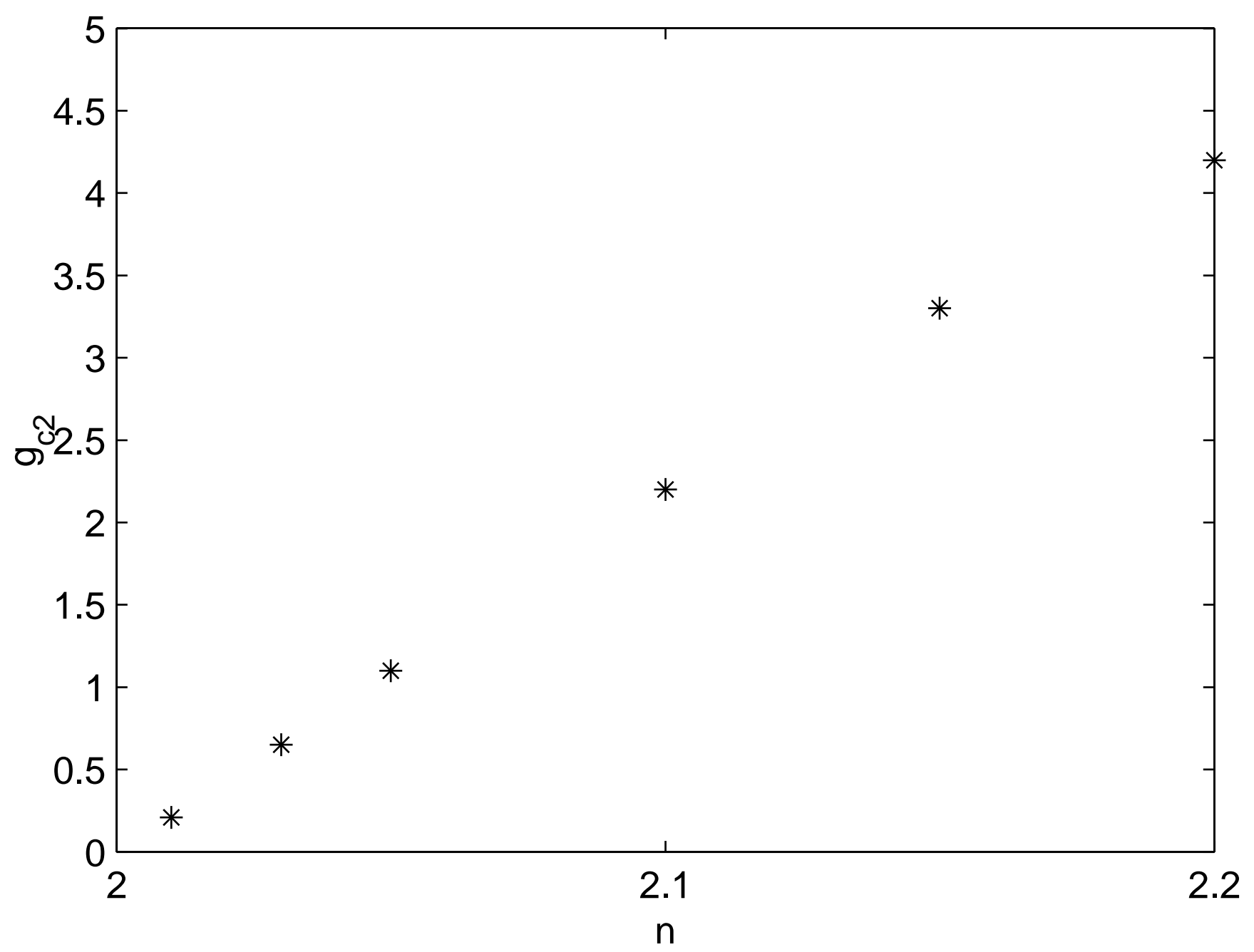

FIG. 3: Critical coupling for the stability of doubly quantized vortices, $g_{\mathrm{c} 2}$, as a function of the power $n$ for a condensate in a power-law trap. The analysis of Sec. II predicts that the curve goes to zero when the trapping power approaches 2 , in accordance with these numerical results.

10-1 and 10-8 and between 11-1 and 11-8. On the other hand, the configurations 9-7, 10-8 and 11-8 have entirely different symmetries from 9-8, 10-9 and 11-9, respectively, and those transitions are discontinuous. Finally, the three last-mentioned states transform smoothly into arrays of singly quantized vortices (9-9, 10-10 and 11-11) when $g$ increases, by splitting of the central vortex into singly quantized ones. Figure 6 illustrates this.

\section{CONCLUSIONS}

We have determined the criteria for existence of vortices with circulation quantum numbers larger than unity in rotated trapped Bose-Einstein condensates. We have proven that in both two- and three-dimensional systems, multiply quantized vortices are the energy minimum if the trapping potential in the plane perpendicular to the axis of rotation is steeper than harmonic, and the interaction is sufficiently weak so that the vortex cores are not much smaller than the cloud, and the external rotation frequency is within appropriate limits. For stronger interactions, the multiply quantized vortices break up into arrays of several vortices. The physics of the transition is the interplay between the inter-vortex repulsion and the trapping potential. In the case of two-dimensional systems, we have numerically determined the regimes of thermodynamic stability of different vortex configurations. For clouds rotated at large angular velocities, there are regimes where arrays containing both singly and multiply quantized vortices are stable.

Most trapping potentials used in experiments are harmonic, but using Laguerre-Gaussian beams one can construct optical traps of arbitrary even power laws, where our predictions can be tested [19]. A two-dimensional Sodium 


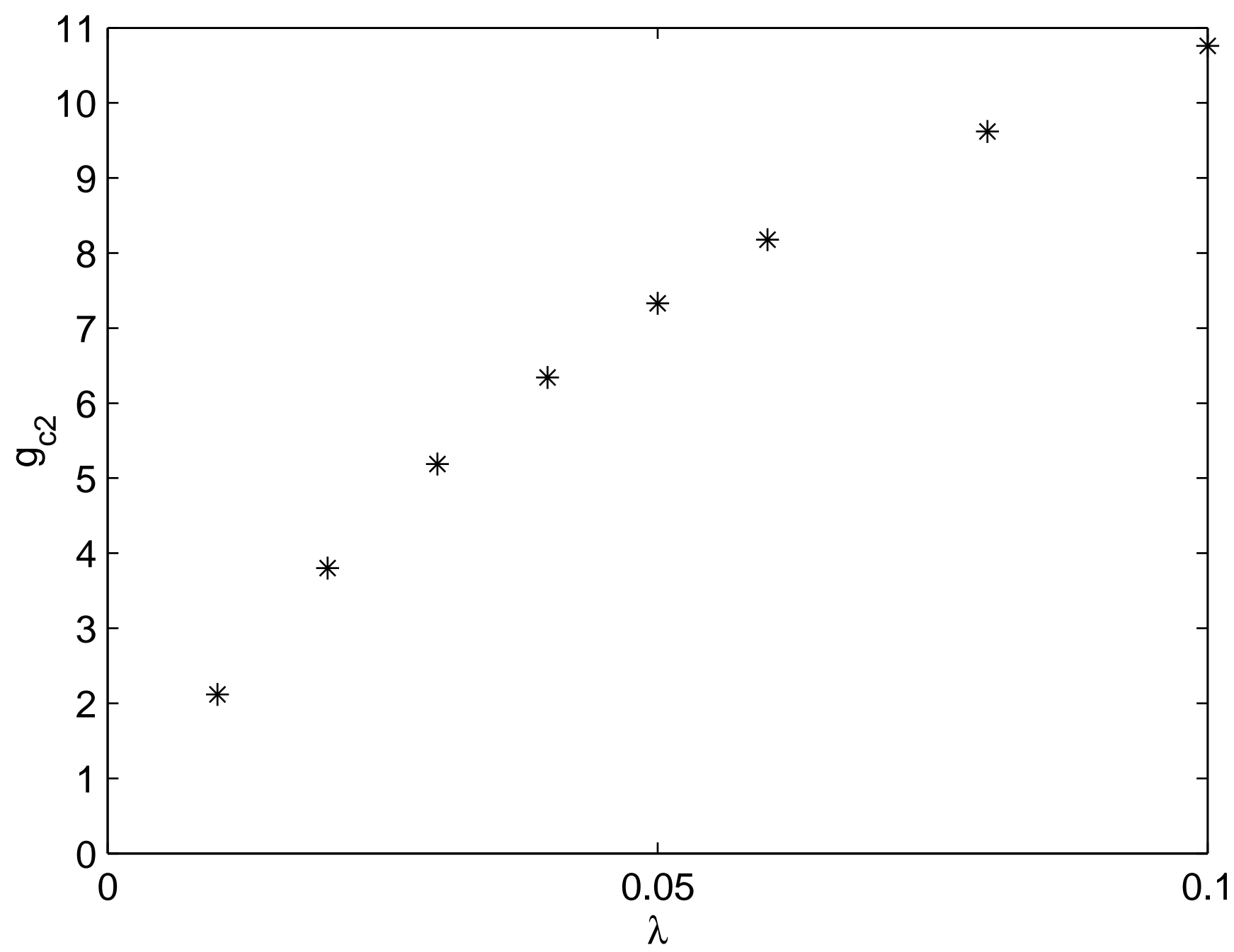

FIG. 4: Critical coupling $g_{\mathrm{c} 2}$ for a condensate trapped in an anharmonic potential as a function of the coefficient $\lambda$ of the quartic term. When $\lambda$ approaches zero, the trap approaches the harmonic case, and the critical coupling drops to zero.

condensate can host doubly quantized vortices if the number of particles is of the order 1000 or smaller, and a trap anharmonicity of about $10 \%$ is introduced. Alternatively, the appropriate weak-coupling regime can be attained by using Feshbach resonances to diminish the scattering length [20]. In a three-dimensional system, a quantitative determinition of the conditions has been prohibited by numerical limitations. The technique of vortex imaging by interference [21], which directly images the phase of the condensate, promises to be a convenient way to detect multiply quantized vortices. Alternatively, one can look for multiply quantized vortices by measuring the angular momentum of the system [22], or simply by looking for anomalously large vortex cores.

\section{Acknowledgments}

I am grateful to my supervisor Jørgen Rammer for supervising this work, and Ping Ao for supervision at the early stages. I would also like to thank Georgios Kavoulakis, C. J. Pethick, Thomas Busch and Lars Melwyn Jensen for 


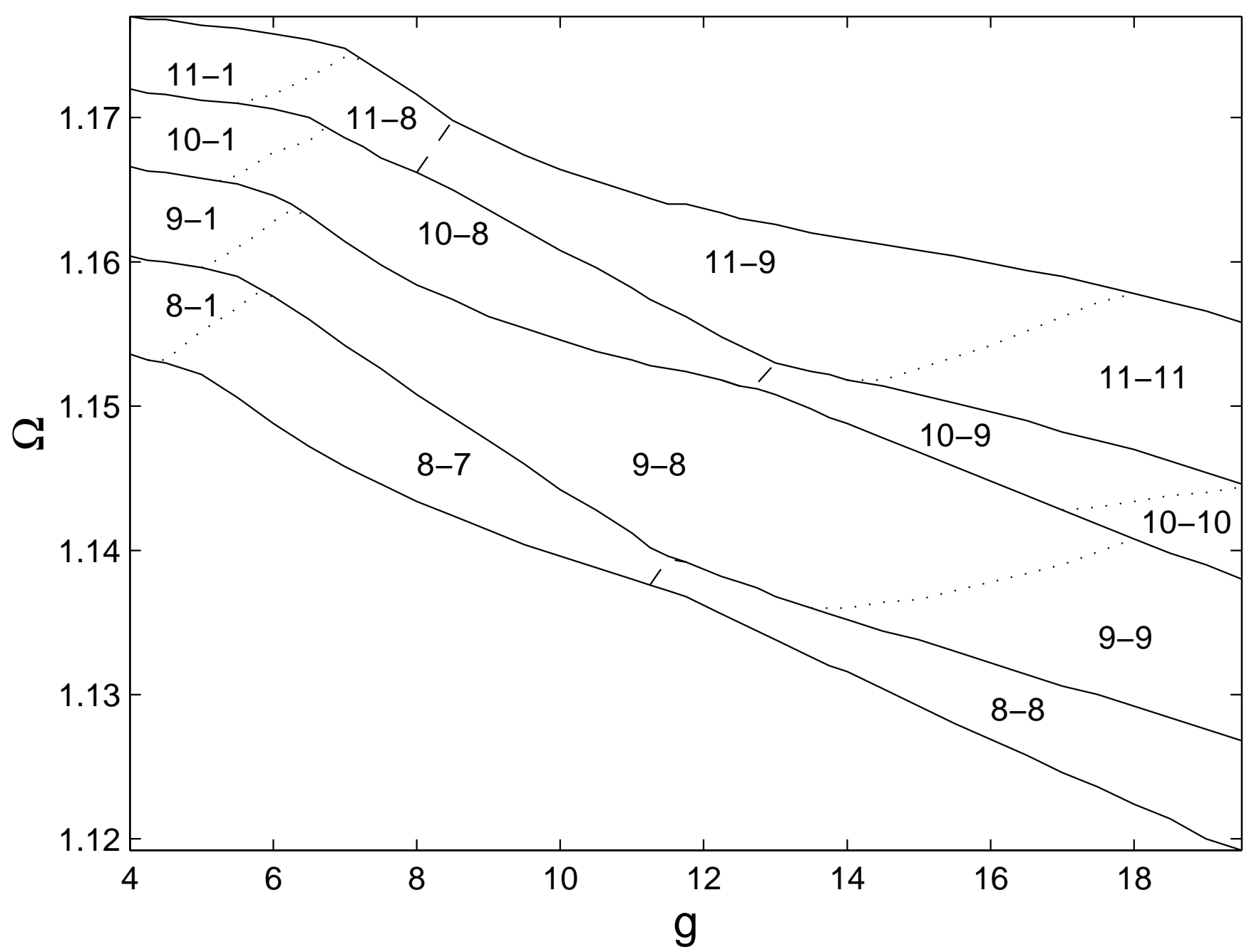

FIG. 5: Phase diagram for a rotated condensate in a power-law trap of power $n=2.2$. Full lines indicate the critical frequencies separating states of different total circulation. Dashed lines mark discrete transitions between states of different symmetry but equal total circulation. Dotted lines mark continuous transitions between states of equal total circulation but different number of singularities. Only the critical lines for circulation $q$ between 8 and 11 are shown. The different phases are labelled by their total circulation, followed by the number of singularities in the system; phases such as 8-7 thus contain both singly and multiply quantized vortices in regular patterns.

valuable discussions, NORDITA for travel support and lodging, and Jani Martikainen for help with the numerics.

[1] M. R. Matthews, B. P. Anderson, P. C. Haljan, D. S. Hall, C. E. Wieman, and E. A. Cornell, Phys. Rev. Lett. 83, 2498 (1999).

[2] K. W. Madison, F. Chevy, W. Wohlleben, and J. Dalibard, Phys. Rev. Lett. 84, 806 (2000).

[3] K. W. Madison, F. Chevy, W. Wohlleben, and J. Dalibard, J. Mod. Opt. 47, 2715 (2000).

[4] J. R. Abo-Shaeer, C. Raman, J. M. Vogels, and W. Ketterle, Science 292, 476 (2001).

[5] R. J. Donnelly, Quantized vortices in helium II (Cambridge University Press, Cambridge, 1991).

[6] M. Baert, V. V. Metlushko, R. Jonckheere, V. V. Moshchalkov, and Y. Bruynseraede, Phys. Rev. Lett. 74, 3269 (1995).

[7] R. Blaauwgeers, V. B. Eltsov, M. Krusius, J. J. Ruohio, R. Schanen, and G. E. Volovik, Nature 404, 471 (2000).

[8] V. A. Schweigert, F. M. Peeters, and P. Singha Deo, Phys. Rev. Lett. 81, 2783 (1998).

[9] G. M. Kavoulakis, B. Mottelson, and C. J. Pethick, Phys. Rev. A 62, 63605 (2000).

[10] D. A. Butts and D. S. Rokhsar, Nature 397, 327 (1999).

[11] Y. Castin and R. Dum, Eur. Phys. J. D 7, 399-412 (1999).

[12] E. P. Gross, Nuovo Cimento 20, 454 (1961); J. Math. Phys. 4, 195 (1963). 

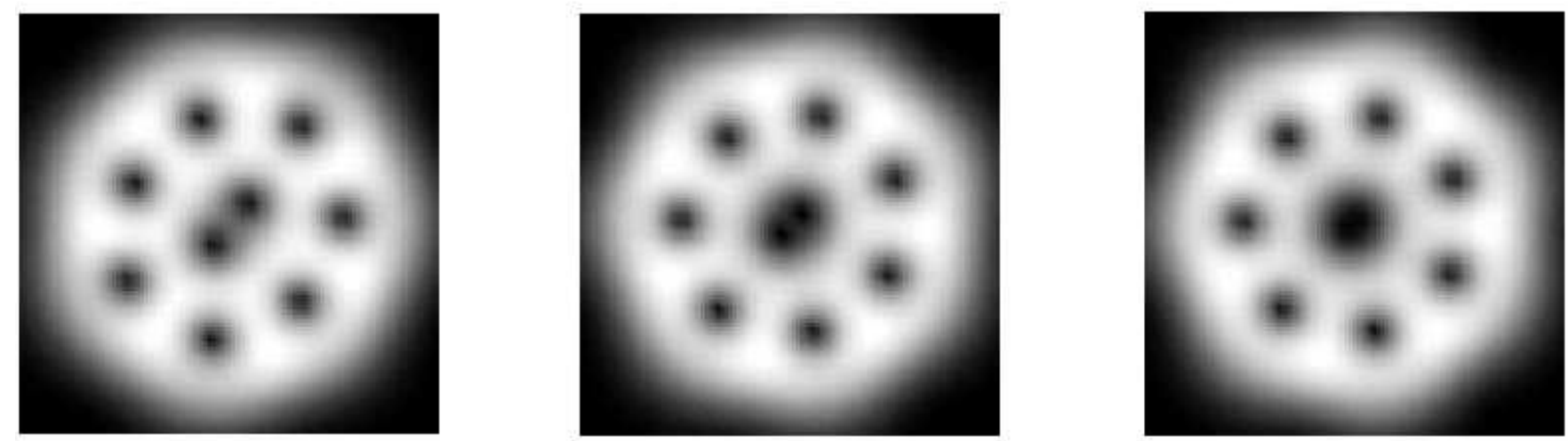

FIG. 6: Density plots for a two-dimensional condensate in a power-law trap of power 2.2, at coupling $g=19.5$. The leftmost panel is the minimum-energy configuration for rotation frequency $\Omega=1.1300 \omega$, the middle panel shows $\Omega=1.1400 \omega$ and the right panel has $\Omega=1.1460 \omega$. All three states have a total circulation $q=9$. The pictures show that the transition between the phases 9-9 (left and middle panels) and 9-8 (right panel) is continuous.

[13] L. P. Pitaevskii, Zh. Eksp. Teor. Fiz. 40, 646 (1961) [Sov. Phys. JETP 13, 451 (1961)].

[14] H. Smith and C. J. Pethick, Bose-Einstein Condensation in Dilute Gases (Cambridge University Press, to be published).

[15] Emil Lundh, C. J. Pethick, and H. Smith, Phys. Rev. A 58, 4816 (1998).

[16] G. Baym and C. J. Pethick, Phys. Rev. Lett. 76, 6 (1996).

[17] C. J. Pethick, private communication.

[18] A. Görlitz, J. M. Vogels, A. E. Leanhardt, C. Raman, T. L. Gustavson, J. R. Abo-Shaeer, A. P. Chikkatur, S. Gupta, S. Inouye, T. P. Rosenband, D. E. Pritchard, and W. Ketterle, e-print cond-mat/0104549.

[19] T. Kuga, Y. Torii, N. Shiokawa, T. Hirano, Y. Shimizu, and H. Sasada, Phys. Rev. Lett. 78, 4713 (1997).

[20] S. Inouye, M. R. Andrews, J. Stenger, H.-J. Miesner, D. M. Stamper-Kurn, and W. Ketterle, Nature 392, 151-154 (1998).

[21] S. Inouye, S. Gupta, T. Rosenband, A. P. Chikkatur, A. Görlitz, T. L. Gustavson, A. E. Leanhardt, D. E. Pritchard, and W. Ketterle, e-print cond-mat/0104444.

[22] F. Chevy, K. W. Madison, and J. Dalibard, Phys. Rev. Lett. 85, 2223 (2000). 